This is a post-peer-review, pre-copyedit version of a paper published in Kurbanoğlu S, Boustany J, Špiranec S, Grassian E, Mizrachi D \& Roy L (eds.) Information Literacy in the Workplace.

Communications in Computer and Information Science, 810. ECIL 2017: European Conference on Information Literacy, Saint-Malo, France, 18.09.2017-21.09.2017. Cham, Switzerland: Springer Verlag, pp. 409-418. The final authenticated version is available online at:

\title{
Developing information literacy in dependent and disadvantaged circumstances: a transitional approach in the digital health context
}

\author{
Steven Buchanan, Cara Jardine, and Ian Ruthven \\ Department of Computer and Information Sciences, University of Strathclyde, \\ Glasgow UK \\ \{steven.buchanan, cara.jardine, ian.ruthven\}@ strath.ac.uk
}

\begin{abstract}
The purpose of this paper is to advance our understanding of approaches to information literacy skills development in disadvantaged and dependent circumstances, and the role of information behaviour investigations in informing meaningful interventions in context. We report on work with young mothers $(<25)$ from areas of multiple deprivation, seeking to better understand their information needs, and the factors influencing their selective and infrequent engagement with state and voluntary sector information services. We evidence limited progress in addressing digital divide issues, and report young mothers as having multiple and complex information needs, and a dependency upon human information intermediaries due to access and behavioural issues. We discuss design and trial of a tailored digital resource to encourage digital access and use, and in turn literacy development; and report that while found useful by young mothers, usage is likely to be low without human intermediary led intervention. A transitional approach is discussed.
\end{abstract}

Keywords: Information literacy, information behaviour, digital health.

\section{Introduction}

The purpose of this paper is to advance our understanding of approaches to information literacy development in disadvantaged (socio-economic) and dependent (support) circumstances. We report on work with young mothers (age <25) from areas of multiple deprivations (e.g. employment, education, health, crime), seeking to better understand their information behaviours, and the factors influencing their selective and infrequent engagement with state and voluntary sector information services and resources. Identifying a persistent digital divide amongst a high-risk group (health and wellbeing) requiring immediate intervention, we report on the development of a digital resource to provide tailored access to trusted online sources to encourage digital access and use, and in turn, facilitate development of information literacy skills. Our approach to intervention is guided by Eisenberg [1], who reminds us that for information literacy programs to be successful they must be meaningful, and highlights three key interwoven components: tasks based on real needs, 
technology in context, and appropriate information process. In practice, this raised three key research questions:

1. What are the information needs of young disadvantaged mothers?

2. What are the appropriate digital sources to meet these information needs?

3. What would be an appropriate design and approach to digital access and use?

The work responds to calls for "person-based" and "persuasive" approaches to digital health interventions [2], and recognition that "more work is needed to create successful [digital health] engagement strategies" [3, p.1]. In particular, we illustrate the role of information behaviour investigations in informing meaningful interventions in the problematic context.

Our overarching theoretical framework brought together theories and models of information behaviour and social capital to better understand shared aspects of social integration (understood as access, participation, engagement) in the socio-economic context.

\section{Background}

The United Kingdom (UK) has one of the highest rates of teenage pregnancy in Western Europe, with associated conception rates correlated to multiple deprivation indexes. At risk groups are disadvantaged and disengaged, with significant health and wellbeing issues reported for both mother and child. In a review of research studies, Trivedi et al [4] highlights at risk individuals as those: in care; homeless; school underperformers/truants; children of teenage mothers; and from deprived areas. Young mothers are more likely to be single parents, to have experienced family conflict/trauma, not to be in employment, education, or training, and to be at risk of short and long-term mental health issues. Infant mortality rates are higher than those for older women [5], and babies are at greater risk of poor nutrition and childcare [6], and impaired development [7]. Stress and anxiety are heightened amongst young mothers, as are rates of depression [8]. Low literacy levels are also reported [9].

Beyond parenting related information needs, on-going broader support is considered key to long-term success and social mobility [10-11]; however, a systematic review of the research evidence relating to teenage pregnancy, parenting and social exclusion reports that mainstream services "have often failed to provide the holistic care required to support social inclusion or social re-integration for teenage parents" [12, p.4], and in particular, to meet diverse needs and preferences. A key recommendation highlighted the need for tailored information, advice and support [12]. A UK National Health Service (NHS) review of midwife services [13] highlights similar issues, identifying a lack of age appropriate information, unmet social information needs, and issues of equity of access to information. There have been further followon calls for tailored health information [14-15] and increased digital provision [16], reflecting wider concerns regarding limited online information provision tailored to 
young people [17]. However, our work examining the information behaviours of first time mothers [18-19] suggests limited progress in addressing such issues, and reports multiple, complex, and at times competing information needs. Louden et al [18] interviewed 22 first-time general population mothers with an average age of 29, and identified information needs relating to: sleeping, milk feeding, weaning, health and infant care, family, activities, and products. Buchanan et al [19] interviewed 49 professional support workers (family nurses, health visitors, social workers, and voluntary sector support group leaders) working with young mothers $(<25)$ from areas of multiple deprivation, and identified information needs relating to: pregnancy, baby care, finance, family relationships, domestic abuse, education and employment, parent health, and housing. In relation to this, multiple needs could be interrelated, often compounding issues of information overload [19]. For example, financial needs routinely encompassed helping mothers with accessing and understanding state benefits, maternity grants and food and vitamin voucher entitlements; and were often closely linked to housing needs and the fundamental need to provide their child with a habitable home that in situations of sub-standard accommodation or homelessness could present significant health needs for both mother and child.

Low literacy levels (reading and information) are also reported [19], discouraging many mothers from seeking information themselves, and limiting their access to and use of textual information. Issues of misinformation from the use of unreliable sources are reported, as are issues of information comprehension [19]. Practical access barriers are also reported, with many young mothers not possessing a computer and those that do often not able to afford and/or eligible for home internet, and while smartphones are more common, usage can be limited by prepaid credit [19]. Access (and support) is available via public libraries, but few young mothers from areas of multiple deprivations attend library-based groups due to issues of confidence and stigma, which also affects engagement with formal learning programmes more broadly [19]. In such circumstances, many young mothers are dependent upon support workers (e.g. family nurses, health visitors, social workers, voluntary sector support group leaders) as human information intermediaries, particularly when seeking information from external (non-interpersonal and/or digital) sources [19].

In summary, a persistent digital divide is evident amongst a high-risk group with an immediate need for meaningful and persuasive intervention in the problematic context. Reasoning that a formal information literacy program was unlikely to succeed due to complex access and internalised behavioural barriers, our first priority was to develop a tailored digital resource that might encourage digital access and use, and that in turn, might facilitate information literacy development.

\section{Methodology}

A typology of information needs (research question one (RQ1)) was developed from synthesis of information needs data from previous studies [18-19] discussed above, with meaningful categories identified via cross-study thematic analysis and iterative 
pattern coding [20]. Periodic team-based code checking was conducted to check developing categories for coherence, consistence, and distinctiveness [21]. Categorised needs then specified the information services to be provided by the digital resource, and the menu elements of the graphical user interface (GUI).

Appropriate online information sources (RQ2) were identified via service directories and online searches (identified needs providing search parameters), and website content analysis, approached in a systematic and structured manner via selective identification of UK non-commercial state and voluntary sector websites, followed by top-down content analysis of respective webpages. Such an approach has been previously employed [22] to identify digital services and was tailorable to study search parameters. Commercial sites were not included for reasons of trust amongst mothers [18]. Redundant sites (mirrored content and/or link) were also removed. Shortlisted sites were mapped via a matrix to identified information needs, and assigned one or more of the following values: 3. Provides parent tailored information, 2. Is a primary source of information (one or more information needs), 1. Is a secondary source of some information (one or more information needs). The best matches combined $3 \& 2$ (i.e., a primary source of parent-tailored information for one or more information needs). Selected sites then provided the underlying architecture (information sources) for the digital resource.

To explore design and use of the digital resource (RQ3), individual and small group field trials and discussions were held with young mothers. A purposive approach to sampling defined the key participant inclusion criteria as being a mother aged <25 from an area of multiple deprivation as defined by the Scottish Index of Multiple Deprivation $^{1}$, with participants recruited via voluntary sector support groups and the UK National Health Service Family Nurse Partnership (FNP) programme. The resource was described to participants as a resource to provide mothers with tailored access to trusted online information, with the sessions framed as open exploratory discussions to solicit feedback and contribute to further development of the resource.

Ethical approval was obtained via Institutional Ethics Committee, with all experiments run in strict accordance with the guidelines set out by our Institutional Code of Practice on Investigations of Human Beings.

\section{Findings}

In presenting and discussing our findings we return to our research questions.

\subsection{What are the information needs of young disadvantaged mothers?}

Synthesis of information needs data from previous studies discussed above [18-19] identified the following information needs amongst young disadvantaged mothers:

\footnotetext{
${ }^{1}$ http://www.gov.scot/Topics/Statistics/SIMD
} 
pregnancy; labour and birth; child development; child health; parent health; playtime activities; childcare; family relationships; housing; money; legal advice; work, education and training; domestic abuse; stress; and helplines. It is notable that, in comparison to the needs of older (avg. age 29) general population mothers, the needs of young $(<25)$ disadvantaged mothers extend beyond parenting to individual issues of poverty and personal development. A high level of element (information need) interactivity is also evident in reports of interrelated needs [19], which is known to be cognitively demanding, requiring systematic and/or simplified learning processes to understand [23], and influencing later design decisions.

\subsection{What are the appropriate digital resources to meet these information needs?}

In identifying appropriate online sources to meet identified information needs, an initial list of 98 candidates (websites) was reduced to 36 when redundant sites were removed (a step to simplify design and the user experience, and in particular, reduce cognitive load). Beyond identifying primary sources of information for parents, this step also identified gaps in provision; most notably to meet housing information needs, with the most appropriate source of information being within the voluntary sector rather than the responsible local (state) authority. Once primary online sources were identified for each information need, this formed the initial architecture of the prototype with eight of the fifteen identified websites parent-tailored. During content analysis and mapping, initial child and parent health categories (i.e., pregnancy, labour and birth, child development, child health, parent health) were noted to be logically grouped on existing websites separated by child age (i.e., baby or toddler), and this convention was adopted in our own model, and a general health category also added (for general health needs). In consideration of low literacy and potentially complex and problematic situations, three further categories of additional support were also added: a-z of common terms, helplines, and additional links.

\subsection{What would be an appropriate design and approach to digital access and use?}

The graphical user interface (GUI) was designed to intentionally mirror ubiquitous and widely accepted tablet and smartphone design (see Figure 1). Cognisant to issues of literacy and cognitive load [19], we considered it beneficial to begin with a GUI design with minimal text, and a simplified architecture that mitigated for issues of information overload common in the initiation and selection stages of information seeking [24] via meaningful (needs based) search processes that expedited access to a single primary source of trusted online information for each category of need. In psychology and cognitive science our categories of need can be considered as schemas, which "can be treated as a single element in working memory and thus heavily decrease cognitive load associated with the performance of later tasks" [25, p.87]. Associated text (category titles) scored 6 in a Flesh-Kincaid readability test indicative of general readability by the adult population. In Big6 terms, we sought to simplify initial steps of task definition, information seeking, location and access. 
12 young mothers (aged 15-23) variously attending third sector parent support groups or part of the NHS FNP programme volunteered to provide feedback (via individual and small group sessions). Comments regarding design were mixed. Single direct links to trusted resources were positively commented on by several mothers, and felt to avoid "unnecessary" and "tedious" navigation, and providing "guarantees the information is reliable". However, several mothers also described the design as "plain" and "boring", and suggested that there could be "a more interesting background" and in relation, that it "could be a bit more colourful and not just white". Positive pictures of parenthood were also suggested. Some mothers also suggested having a page beneath each icon providing more links for each topic and additional context and direction such as "general summaries" of the information available beneath.

\section{Mothers Digital Gateway}

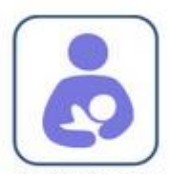

My Baby and Me
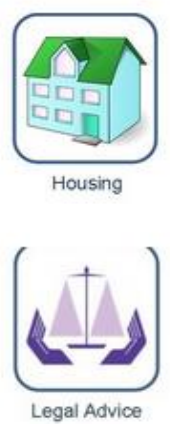

Legal Advice
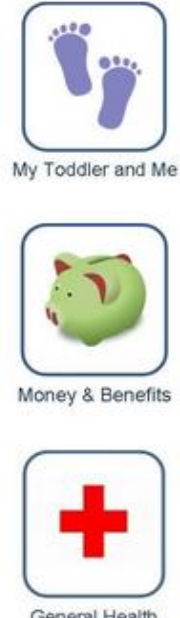

General Health

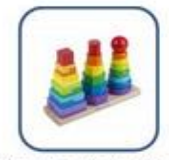

Playtme Tips \& Ideas
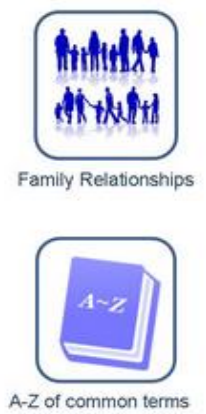
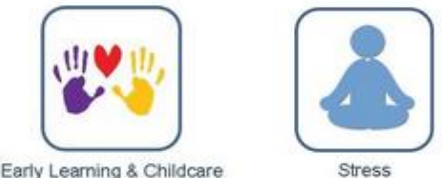

Stress
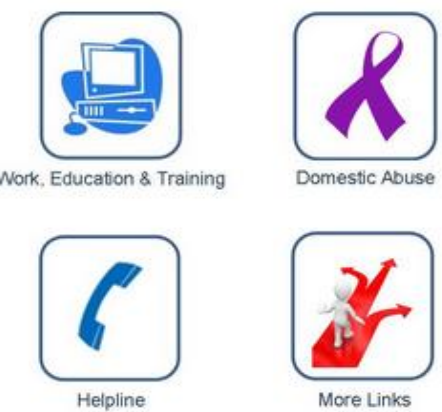

Fig. 1. Prototype GUI design ${ }^{2}$

Some mothers disliked the prototype name ('Mothers Digital Gateway'). It was suggested that "mother" needs to be replaced with a gender neutral term encompassing fathers and carers, and that "gateway" might be replaced with "help book", "helpful info for parents" or "survival guide", although with the latter, when it was mentioned that there already exists a publication titled 'The Young Parents Survival Guide', another participant considered this insulting as she felt it suggests that young people cannot cope with parenthood, and in relation, another commented that, "when you get called a young mum or a young parent, it kind of makes you feel

\footnotetext{
${ }^{2}$ The launched version of this prototype can be accessed at: http://yoyo.cis.strath.ac.uk/
} 
like you're less... because of the way the generations seen as teenagers or whatever... or making you seem less valuable as a parent than older mums". This is an important consideration as stigma is a known issue amongst young mothers [26], with stigma also known to influence information practices [27]. Comments highlight sensitive issues of self-identity, and remind us of the importance of positive constructive communication in progressive situations (i.e., parenthood).

With regard to utility, mothers appeared to intuitively understand the resources' purpose and provided positive feedback regarding categorised information need topics, with none of the topics questioned and three further suggestions made: sexual health, local information and useful phone numbers. Several indicated that they might use the resource with simplified access appearing valued. For example, one mother while browsing linked sites underneath topics commented that without such a resource it can "take forever" to find trusted sites, and another that it was "good to have all the trustworthy sites in one place". Mothers also thought that it should have its own domain with visible links from NHS and public library sites (note: the prototype was located on the parent research project website during these trials).

The trial in itself could also be seen to increase awareness of online resources amongst several participants. For example, one mother while exploring the website link under family relationships commented, "I quite like this one - family lives - I've never seen that one before". However, the majority of participants as part of general discussions also indicated a low use of online sources (favouring interpersonal sources such as family and health visitors instead), and infrequent use of online resources beyond Google. For example, the majority of our participants were unaware of ReadySteadyBaby (NHS Scotland's main online guide to pregnancy and the first 12 months of parenthood), and whilst all knew of Young Scot (Scotland's national information and citizenship resource for young people aged 11-25), none used the website or knew of any peers who did, with the resource described by one as being "not really a thing that young people are actually interested in". In relation, one participant commented more broadly, 'I don't think many people look online for information', a comment supported by another who replied 'I know I don't'.

\section{Discussion}

Young mothers from areas of multiple deprivations have multiple, complex, interwoven, and at times competing information needs, extending beyond parenting needs shared with general population peers, to individual issues of poverty and personal development. Our findings highlight the importance of situational understanding of information needs, and the potential risks involved in assumptions of homogeneity. Attempting to provide holistic support in such complex circumstances has been a significant and enduring challenge to date with repeated calls for tailored digital support largely unmet. We have demonstrated the role of information behaviour investigations in identifying information needs, and learning needs; to inform meaningful interventions in the problematic context, and in particular, to 
develop a tailored resource to facilitate and encourage digital access and use. Importantly, given issues of cognitive load, identified categories of information need can also act as schemas to reduce cognitive load during information seeking activity, helping to simplify initial tasks of location and access, and facilitating systematic approaches to information seeking in instances of multiple needs with high element (need) interactivity.

In pilot trials, our young mothers appeared to intuitively understand and interact with the resource, providing positive feedback regarding usability and usefulness, but our findings also suggest that usage is likely to be extremely low by itself. Returning to Eisenberg's [1] three components of effective IL programs, the resource is clearly focused on real information needs, and in meeting those needs helps place technology in purposeful context. It is the third component, process, that requires careful consideration, having to overcome complex access and behavioural issues, the former influenced by digital divide issues, the latter by socio-psychological factors. Given these issues, it is evident that interventions will need to begin in the physical space, and be human intermediary led, which raises further questions of who and how?

We know that young mothers mostly do not use libraries or librarians; but that their support workers (family nurses, health visitors, social workers, voluntary sector support group leaders), beyond being a key source of information in themselves, play a key role in directing and linking young mothers to other information services, groups and resources, and dealing with misinformation [19]. Support workers, as information intermediaries, are therefore ideally placed to utilise the digital resource in their everyday interactions with young mothers, providing access to, and encouraging use of trusted sources of online information, and providing opportunity to discuss and develop information literacy skills in a practical interactive manner. In combination (resource and intermediary), a transitional approach can be applied in the everyday context. However, to be successful, careful consideration has to be given to both the meeting of immediate needs, and longer-term transitions to self-efficacy.

In the learning context, Eisenberg recommends 'continually working with users to help them recognize where they are in the process' [1, p.43]. This of course requires that there be an agreed upon and understood information process in place that tasks and actions can be related to, and that encourages reflective practice, and develops metacognitive skills. For our purposes such an information process must be adaptable to semi-structured everyday situations, and implementable by non-information professionals, such as nurses, and is reserved for the next stage of this research as part of field trials of the resource involving NHS family nurses providing outreach support to young disadvantaged mothers.

\section{Conclusion}

A persistent digital divide is evident amongst young mothers from areas of multiple deprivations, who have multiple, complex, interwoven, and at times competing 
information needs, extending beyond parenting needs to issues of poverty and personal development. There are complex access and behavioural issues to consider, the former influenced by digital divide issues, the latter by socio-psychological factors. Providing support in such circumstances has been a significant and enduring challenge to date.

We have demonstrated the role of information behaviour investigations in identifying information needs, and learning needs, to inform meaningful interventions, and in particular, to develop a tailored digital resource to facilitate and encourage digital access and use in the problematic context. However, while found to provide meaningful and useful access to trusted online information for young mothers, usage is likely to be low without purposeful human intermediary intervention.

The resource can be used by human intermediaries to direct young mothers to tailored and trusted online sources of information, and as a tool to discuss and develop information literacy skills in an interactive and practical manner, developing selfefficacy over time. This requires an information process to be in place that encourages reflective practice and the development of metacognitive skills. It must also be adaptable to semi-structured everyday situations, and implementable by noninformation professionals. In combination (resource, intermediary, process), a holistic transitional approach to intervention can then be taken in the problematic context.

\section{References}

1. Eisenberg, M.B.: Information literacy: Essential skills for the information age. DESIDOC Journal of Library \& Information Technology, 28(2), 39-47 (2008)

2. Yardley, L., Morrison, L., Bradbury, K. and Muller, I.: The person-based approach to intervention development: application to digital health-related behavior change interventions. Journal of medical Internet research, 17(1), 30 (2015)

3. O’Connor, S., Hanlon, P., O'Donnell, C.A., Garcia, S., Glanville, J. and Mair, F.S.: Understanding factors affecting patient and public engagement and recruitment to digital health interventions: a systematic review of qualitative studies. BMC medical informatics and decision making, 16(1), 120 (2016)

4. Trivedi et al.: Update on the Review of Reviews on Teenage Pregnancy and parenthood. Centre for Research in Primary and Community Care, http://www.nice.org.uk/nicemedia/pdf/TeenagePregnancyUpdateReviewFeb08.pdf (2007)

5. Torvie, A. J., L. S. Callegari, et al.: Labor and delivery outcomes among young adolescents. American journal of obstetrics and gynecology 213(1): 95, 91-95 (2015)

6. Harron, K., Gilbert, R., Cromwell, D., Oddie, S., \& van der Meulen, J.: Adversity and risk of poor birth and infant outcomes for young mothers: a population-based data-linkage cohort study. The Lancet, 388, S54 (2016)

7. UNICEF: Child poverty in perspective: an overview of child well-being in rich countries. Innocenti Report Card 7(2), (2007)

8. Raskin, M., Easterbrooks, M.A., Lamoreau, R.S., Kotake, C., \& Goldberg, J.: Depression Trajectories of Antenatally Depressed and Nondepressed Young Mothers: Implications for Child Socioemotional Development. Women's Health Issues, 26(3), 344-350, (2016) 
9. Bennett, I. M., Frasso, R., Bellamy, S. L., Wortham, S., \& Gross, K. S.: Pre-teen literacy and subsequent teenage childbearing in a US population. Contraception 87(4), 459-464, (2013)

10. Smith, D.M., and Roberts, R.: Social inequality and young pregnancy: the causal attributions of young parents in London, UK. Health Place 15(5), 1054-60, (2011)

11. Smith, M. L., Gilmer, M. H., Salge, L. E., Dickerson, J. B., \& Wilson, K. L.: Who Enrolls in Teen Parent Education Programs? An Emphasis on Personal and Familial Characteristics and Services Received. Child and Adolescent Social Work Journal, 1-16, (2012)

12. Harden, A., Brunton, G., Fletcher, A., Oakley, A., Burchett, H., \& Backhans, M.: Young people, pregnancy and social exclusion: A systematic synthesis of research evidence to identify effective, appropriate and promising approaches for prevention and support. EPPICentre, Social Science Research Unit, Institute of Education, University of London, (2006)

13. Coltart, L.: Meeting the needs of pregnant teenagers across NHS Greater Glasgow \& Clyde, http://health.cat/ref/pdf.php?search=NHS\%20Greater\%20Glasgow\%20and\%20Clyde, (2007)

14. Berkule-Silberman, S. B., Dreyer, B. P., Huberman, H. S., Klass, P. E., \& Mendelsohn, A. L.: Sources of parenting information in Low SES Mothers. Clinical pediatrics 49(6), 560$568,(2010)$

15. Hagell, A., and Coleman, J.: Adolescent health in the UK today: where next. AYPH, http://www.ayph.org.uk/publications/278_Adol\%20health\%20in\%20the\%20UK\%20today \%2019\%20June\%202012.pdf, (2012)

16. Joint Strategic Needs Assessment Under-18 conceptions in the Lancashire sub-region 2011/12, http://www.lancashire.gov.uk/corporate/web/?siteid=6107\&pageid=35232\&e=e, (2012)

17. Reading Agency: Libraries and digital: research into the use of digital media in libraries to develop audiences for reading, www.readingagency.org.uk/, (2011)

18. Louden, L., Buchanan, S. and Ruthven, I.: The everyday life information seeking behaviours of first-time mothers, Journal of Documentation, 72 (1), 24 - 46, (2016)

19. Buchanan, S., Jardine, C., \& Ruthven, I.: Information behaviours in disadvantaged and dependent circumstances and the role of information intermediaries. Forthcoming, (2017)

20. Miles, M., \& Huberman, A.: Qualitative data analysis: An expanded sourcebook. Sage, (1994)

21. Braun, V., \& Clarke, V.: Using thematic analysis in psychology. Qualitative research in psychology, 3(2), 77-101, (2006)

22. Buchanan, S., and McMenemy, D.: Towards a public library digital service taxonomy, European Conference Digital Libraries (ECDL 2010): Research and Advanced Technology for Digital Libraries Lecture Notes in Computer Science, 425-428, (2010)

23. Paas, F., Renkl, A., and Sweller, J.: Cognitive load theory and instructional design: Recent developments. Educational psychologist, 38(1),1-4, (2003)

24. Kuhlthau, C.C.: Seeking meaning: A process approach to library and information services. Libraries Unltd Incorporated, (2004)

25. Van Merriënboer, J.J. and Sweller, J.: Cognitive load theory in health professional education: design principles and strategies. Medical education, 44(1), 85-93, (2010)

26. Ormiston, R., McConville, S., \& Gordon, J.: Evaluation of the Family Nurse Partnership Programme in NHS Lothian, Scotland: $2^{\text {nd }}$ report - late pregnancy and postpartum, http://www.maternal-and-early-years.org.uk/topic/pregnancy/teenage-pregnancy\#evidence, (2012)

27. Lingel, J., \& Boyd. D.: Keep it secret, keep it safe: Information poverty, information norms, and stigma. Journal of the American Society for Information Science and Technology, 64(5), 981-991, (2013) 